\title{
The Effects of Acute Arginine Vasopressin Administration on Social Cognition in Healthy Males
}

\author{
Amanda R. Kenyon, Gail A. Alvares, Ian B. Hickie, and Adam J. Guastella \\ Brain \& Mind Research Institute, The University of Sydney, Sydney, NSW 2050, Australia \\ Correspondence should be addressed to Adam J. Guastella; adam.guastella@sydney.edu.au
}

Received 25 March 2013; Revised 26 April 2013; Accepted 13 May 2013

Academic Editor: Elisabetta Baldi

Copyright ( 2013 Amanda R. Kenyon et al. This is an open access article distributed under the Creative Commons Attribution License, which permits unrestricted use, distribution, and reproduction in any medium, provided the original work is properly cited.

\begin{abstract}
The structurally similar neuropeptides and hormones oxytocin (OT) and arginine vasopressin (AVP) play significant and complex roles in modulating a range of social behaviours, including social recognition and bond formation. Although OT has well-known roles in facilitating prosocial behaviors and enhancing emotion recognition, AVP has received increasing interest for diverging effects on social cognition behaviour most notably in males. The current study aimed to determine whether AVP also modulates the ability to understand emotion. Using a randomised double blind procedure, 45 healthy young males received either an AVP or placebo nasal spray and completed the Reading the Mind in the Eyes Test (RMET). In contrast to previous findings, there were no significant differences observed in performance on the RMET between AVP and placebo groups, even after examining items separated by task difficulty, emotional valence, and gender. This study provides diverging evidence from previous findings and adds to the growing body of research exploring the influence of neuropeptide hormones in social behaviour. It demonstrates that in this sample of participants, AVP does not enhance the ability to understand higher order emotion from others. Implications and suggestions for future AVP administration studies are discussed.
\end{abstract}

\section{Introduction}

A burgeoning amount of research in the field of social neuroscience has highlighted the importance of the neuropeptides oxytocin (OT) and arginine vasopressin (AVP) in the control of mammalian social behaviour [1]. Both peptides are structurally similar, differing by only two amino acids, are synthesised in the hypothalamus, and stored via the posterior pituitary for peripheral and central release [2]. The single OT receptor and three AVP receptors (AVPla, AVP1b, and AVP2) are widely distributed throughout the central and peripheral nervous system [3], particularly in limbic regions. They can additionally act as neuromodulators, influencing neurotransmission. For example, AVP acts on the AVPRlb receptor in the anterior pituitary to enhance cortisol secretion.

Although much research has exclusively focused on the role of OT in social cognition and behavior (for review see [1]), studies in animal models utilising AVP have demonstrated more specific effects on enhancing effects on social memory, or recognition, and bond formation [4-6]. In humans, we previously demonstrated that AVP nasal spray administration enhances recognition of previously seen emotional faces [7] and words [8] in healthy males. Thompson and colleagues showed that AVP enhances facial responses to neutral facial stimuli in men [9] as well as demonstrating sexually dimorphic effects in men and women on affiliative and aggressive facial motor responses to unfamiliar faces of the same sex [10]. Of particular note, neural evidence suggests that intranasal AVP administration alters activity in the temporoparietal junction when processing social information, a key area for theory of mind tasks [11], and potentially alters medial prefrontal cortex connectivity to the amygdala during exposure to emotionally negative faces [12]. Taken together, these findings suggest that intranasal AVP may modulate social cognitive functioning at both behavioral and neural levels.

The role that these neuropeptides play in social cognition is of particular interest, as it has been a key argument for the use of $\mathrm{OT}$ as a therapeutic in psychiatric disorders 
where social cognition deficits are a key characteristic. For example, Domes and colleagues [13] demonstrated that OT administration improves the ability of healthy males to identify emotions from photographs of the eye regions of faces using the Reading the Mind in the Eyes Test (RMET; [14]), a finding that we replicated in male adolescents with autism spectrum disorders [15]. However, it has been noted that AVP and its analogues have affinity across not only the three AVP receptors but also the OT receptor [16]; it may be that the administration of AVP may exert similar behavioural and neural effects to OT in some contexts due to crossreactivity of AVP on the OT receptor.

Recently, Uzefovsky and colleagues [17] examined the effects of intranasal AVP on the recognition of emotions, finding that AVP decreased performance on the RMET; performance was particularly worse under AVP for photos of males or photos depicting more negative emotions. The authors argued that this finding is in line with the effects of AVP on aggression-related social behaviors in males. This divergent finding, compared to previous results from OT administration studies, has significant implications for the use of AVP in decreasing male social cognitive ability and use for these neuropeptides as therapeutics in disorders of social cognitive deficit. We thus aimed to replicate this finding in a sample of healthy adult males.

\section{Materials and Method}

2.1. Participants. Forty-five healthy young adult male students from the University of Sydney (age $=18-60$ years; mean $=21.96$; $\mathrm{SD}=7.05$ ) were randomly assigned in a doubleblind manner to receive either 20 International Units (I.U.) of AVP $(n=21)$ or placebo (PL; $n=24)$ (three participants from the original sample of forty-eight participants [7] did not complete the RMET and were thus not included in any analyses for the present study). Nasal sprays were developed by a compounding chemist (AVP purchased from Link Pharmaceuticals, Australia), with an identical placebo containing all ingredients except the active AVP. Exclusion was determined from a brief self-report interview by a trained experimenter. The exact protocols for inclusion/exclusion criteria, nasal spray administration, and procedures for consent have been reported elsewhere [7]. Briefly, participants were required to be free of any mental or physical illness and refrain from consuming alcohol and caffeine on the day of the experiment and food and drinks (other than water) for two hours prior to the experiment. Ethical approval was provided by the University of Sydney Human Research Ethics Committee (11693), and written consent was obtained from each participant after being provided a full description of the study.

2.2. Materials and Procedure. Questionnaires completed by participants prior to nasal spray administration were the Brief Fear of Negative Evaluation [18], to assess specific social concerns, the Depression, Anxiety, and Stress Scale [19], and the Profile of Mood States (POMS; [20]) to track changes in mood.
After completing questionnaires, participants self-administered a nasal spray (either AVP or placebo); 45 minutes later they completed a second POMS, and a test battery comprising of two recognition tasks $[7,8]$ and the RMET. The RMET [14] assesses one's ability to read emotions from solely the eye regions from photographs of affective facial expressions. It is a widely used test of emotion recognition in healthy $[13]$ and clinical $[14,15]$ populations. The test consists of 36 black-and-white photographs of the eye regions of male and female faces. Participants choose one of four descriptor words (three distracters and one correct) that describe the mental state of the person in the photograph. Stimuli were presented in the same sequence for each participant on a 24 inch computer monitor. Results were calculated as the number of items correct as a percentage of the overall number of items.

The following day, participants self-reported any possible side effects due to drug administration and beliefs about which drug they believed they had received.

\section{Results}

Independent samples $t$-tests revealed no significant differences between AVP and PL groups in age, depression, anxiety, stress, fear of negative evaluation, or any mood subscale; all $P$ values $>0.05$. Chi-squared analyses run on number and type of reported side effects revealed no significant differences between groups (exact details have been previously reported in [7]); $P>0.05$. Additionally, as we have previously reported, most participants believed they had received the placebo spray, with a trend for this belief to be slightly higher in the AVP group [7].

Total performance on the RMET, calculated as percentage correct, did not significantly differ between groups (mean \pm $\mathrm{SD}: \mathrm{AVP}=77.12 \pm 9.29 ; \mathrm{PL}=78.82 \pm 11.26 ; t(43)=0.55 ; P=$ 0.59 ; Cohen's $d=0.16$ ). Items were then divided in smaller subsets according to difficulty (easy or hard; $[13,15]$ ), valence (negative or positive mental state), and gender of photo [17], scored as percentage correct within each subset. Compared to the PL group, AVP did not result in any significant differences when separating items by difficulty, valence, or gender; all $P$ values $>0.05$.

\section{Discussion}

The aim of the present study was to evaluate the effects of intranasal AVP on emotion recognition in healthy males and compare findings to those recently reported by Uzefovsky et al. [17]. In contrast to these findings, we demonstrated no effect of AVP administration on RMET performance; participants in the AVP and PL groups exhibited similar ability to detect emotion from eye regions of faces. This was evident even when examining subset of items that were separated by difficulty, valence, and gender. These findings are also in marked contrast to the noted beneficial effects of OT on social cognition in healthy and autistic males $[13,15]$.

Recent human administration studies have shown that AVP enhances reflexive agonistic facial motor responsiveness 
to same-sex neutral faces in males but affiliative motor responses in females $[9,10]$. We have also previously reported findings in this same sample of participants that AVP administration alters human cognition on tests of emotional memory and word recognition $[7,8]$. Thus, the observed results cannot be due to a failure of the AVP nasal spray to alter other aspects of cognition. Additionally, there were no other differences in task design or study procedure between the present study and the study described by Uzefovsky et al. [17]. It is also worth noting that the original RMET stimuli were not analysed separately by gender or facial expressions. As we have recommended elsewhere [21], further research aiming to specifically explore the effects of gender and different types of facial expression should use an established test battery for these categories that employ full-face photographs. However, given the large effect sizes reported in the latter study (Cohen's $D$ between 0.72 and 0.91 ) and the results we present, the effects of AVP on human social cognition need to be further tested in larger samples to examine the robustness of either finding.

Previous AVP research has argued for a specific regulation of male social behaviour. In comparison to OT, AVP is in greater supply in males and is mainly regulated via interactions with androgen, as well as estrogen, receptor mechanisms [22]. The administration of AVP also appears to have stronger effects in males in animal models [23]. Studies examining genetic polymorphisms in AVP receptors and measuring plasma AVP levels have also shown specific AVP relevant associations with the capacity of male humans to form and maintain relationships $[24,25]$. These findings are particularly of interest considering that disorders of social deficit, like autism spectrum disorders, have dramatically higher prevalence rates in males [26].

Compared to the plethora of OT research in humans (see recent review in [1]), studies examining the effects of AVP administration on subsequent social processes have been more limited. As such, it is more difficult to hypothesise why we did not observe a difference between AVP and placebo groups in emotion recognition performance. However, similar to evidence emerging from OT administration studies, the effects of AVP may be modulated by individual difference and context factors [27]. A recent study examined the effects of basal AVP and exogenous AVP administration on subsequent social cognition in men and women with PTSD [28]. The authors found that men administered AVP demonstrated faster attention to their partner's expression of anger than men who received placebo, with no differences between drugs in women. Interestingly, this effect was specific to partner expressions of anger and not to expressions of anger in a stranger. Thus, the authors concluded that, like OT, AVP does not simply increase the salience of social stimuli, but rather effects are dependent on contextual and individual difference factors. In that study, faster attentional engagement with a partners expressions may represent greater partner bonding; as AVP has been implicated in facilitating pair bonding in rodents [29], these results provided somewhat homologous evidence in humans. Additional factors that may have contributed to increasing variance on emotion recognition performance include personality traits, social context, and genetic polymorphisms of OT and AVP receptor variants [30]. Thus, we suggest that future studies should consider these potential individual difference factors as an additional component to determine AVP efficacy in particular social and emotional tasks, in larger powered studies.

It should be noted that we did not recruit clinical patients with clear social impairments. Our results, therefore, do not directly determine whether AVP does have clinical utility as a treatment for social problem. It should also be noted that we employed a relatively small male sample. Although the sample size was comparable to previous studies in this area $[13,17]$, the large $P$ values observed indicate that there was not any effect of AVP administration and thus could not be attributed to a lack of statistical power. We therefore conclude that, in this sample of male students, AVP does not affect the ability to understand emotions from the eyes of others.

\section{Acknowledgments}

This work was supported by a University of Sydney Bridging Support Grant, an NHMRC Grant (632624; 632625), and an ARC linkage Grant (LP110100513) to A. J. Guastella.

\section{References}

[1] A. Meyer-Lindenberg, G. Domes, P. Kirsch, and M. Heinrichs, "Oxytocin and vasopressin in the human brain: social neuropeptides for translational medicine," Nature Reviews Neuroscience, vol. 12, no. 9, pp. 524-538, 2011.

[2] M. Ludwig and G. Leng, "Dendritic peptide release and peptidedependent behaviours," Nature Reviews Neuroscience, vol. 7, no. 2, pp. 126-136, 2006.

[3] G. Gimpl and F. Fahrenholz, "The oxytocin receptor system: structure, function, and regulation," Physiological Reviews, vol. 81, no. 2, pp. 629-683, 2001.

[4] I. F. Bielsky, S. B. Hu, K. L. Szegda, H. Westphal, and L. J. Young, "Profound impairment in social recognition and reduction in anxiety-like behavior in vasopressin Vla receptor knockout mice," Neuropsychopharmacology, vol. 29, no. 3, pp. 483-493, 2004.

[5] J. N. Ferguson, J. M. Aldag, T. R. Insel, and L. J. Young, "Oxytocin in the medial amygdala is essential for social recognition in the mouse," Journal of Neuroscience, vol. 21, no. 20, pp. 8278 $8285,2001$.

[6] J. T. Winslow, N. Hastings, C. S. Carter, C. R. Harbaugh, and T. R. Insel, "A role for central vasopressin in pair bonding in monogamous prairie voles," Nature, vol. 365, no. 6446, pp. 545548, 1993.

[7] A. J. Guastella, A. R. Kenyon, G. A. Alvares, D. S. Carson, and I. B. Hickie, "Intranasal arginine vasopressin enhances the encoding of happy and angry faces in humans," Biological Psychiatry, vol. 67, no. 12, pp. 1220-1222, 2010.

[8] A. J. Guastella, A. R. Kenyon, C. Unkelbach, G. A. Alvares, and I. B. Hickie, "Arginine Vasopressin selectively enhances recognition of sexual cues in male humans," Psychoneuroendocrinology, vol. 36, no. 2, pp. 294-297, 2011.

[9] R. Thompson, S. Gupta, K. Miller, S. Mills, and S. Orr, "The effects of vasopressin on human facial responses related to social communication," Psychoneuroendocrinology, vol. 29, no. 1, pp. 35-48, 2004. 
[10] R. R. Thompson, K. George, J. C. Walton, S. P. Orr, and J. Benson, "Sex-specific influences of vasopressin on human social communication," Proceedings of the National Academy of Sciences of the United States of America, vol. 103, no. 20, pp. 7889-7894, 2006.

[11] C. F. Zink, L. Kempf, S. Hakimi, C. A. Rainey, J. L. Stein, and A. Meyer-Lindenberg, "Vasopressin modulates social recognitionrelated activity in the left temporoparietal junction in humans," Translational Psychiatry, vol. 1, article e3, 2011.

[12] C. F. Zink, J. L. Stein, L. Kempf, S. Hakimi, and A. MeyerLindenberg, "Vasopressin modulates medial prefrontal cortexamygdala circuitry during emotion processing in humans," Journal of Neuroscience, vol. 30, no. 20, pp. 7017-7022, 2010.

[13] G. Domes, M. Heinrichs, A. Michel, C. Berger, and S. C. Herpertz, "Oxytocin improves "mind-reading" in humans," Biological Psychiatry, vol. 61, pp. 731-733, 2007.

[14] S. Baron-Cohen, S. Wheelwright, J. Hill, Y. Raste, and I. Plumb, "The "reading the mind in the eyes" test revised version: a study with normal adults, and adults with Asperger syndrome or high-functioning autism," Journal of Child Psychology and Psychiatry and Allied Disciplines, vol. 42, no. 2, pp. 241-251, 2001.

[15] A. J. Guastella, S. L. Einfeld, K. M. Gray et al., "Intranasal oxytocin improves emotion recognition for youth with autism spectrum disorders," Biological Psychiatry, vol. 67, no. 7, pp. 692694,2010

[16] M. Manning, A. Misicka, A. Olma et al., "Oxytocin and vasopressin agonists and antagonists as research tools and potential therapeutics," Journal of Neuroendocrinology, vol. 24, no. 4, pp. 609-628, 2012.

[17] F. Uzefovsky, I. Shalev, S. Israel, A. Knafo, and R. P. Ebstein, "Vasopressin selectively impairs emotion recognition in men," Psychoneuroendocrinology, vol. 37, pp. 576-580, 2012.

[18] M. R. Leary, "A brief version of the fear of negative evaluation scale," Personality and Social Psychology Bulletin, vol. 9, no. 3, pp. 371-375, 1983.

[19] P. F. Lovibond and S. H. Lovibond, "The structure of negative emotional states: comparison of the depression anxiety stress scales (DASS) with the Beck Depression and Anxiety Inventories," Behaviour Research and Therapy, vol. 33, no. 3, pp. 335-343, 1995.

[20] D. M. Mcnair, M. Lorr, and L. F. Droppelman, Manual for the Profile of Mood States, Educational and Industrial Testing Service, San Diego, Calif, USA, 1971.

[21] S. Shahrestani, A. H. Kemp, and A. J. Guastella, "The impact of a single administration of intranasal oxytocin on the recognition of basic emotions in humans: a meta-analysis," Neuropsychopharmacology, 2013.

[22] G. J. de Vries, "Sex differences in vasopressin and oxytocin innervation of the brain," Progress in Brain Research, vol. 170, pp. 17-27, 2008.

[23] E. Choleris, A. E. Clipperton-Allen, A. Phan, and M. Kavaliers, "Neuroendocrinology of social information processing in rats and mice," Frontiers in Neuroendocrinology, vol. 30, no. 4, pp. 442-459, 2009.

[24] S. E. Taylor, S. Saphire-Bernstein, and T. E. Seeman, "Are plasma oxytocin in women and plasma vasopressin in men biomarkers of distressed pair-bond relationships?" Psychological Science, vol. 21, no. 1, pp. 3-7, 2010.

[25] H. Walum, L. Westberg, S. Henningsson et al., "Genetic variation in the vasopressin receptor la gene (AVPR1A) associates with pair-bonding behavior in humans," Proceedings of the
National Academy of Sciences of the United States of America, vol. 105, no. 37, pp. 14153-14156, 2008.

[26] M. Yeargin-Allsopp, C. Rice, T. Karapurkar, N. Doernberg, C. Boyle, and C. Murphy, "Prevalence of autism in a US metropolitan area," Journal of the American Medical Association, vol. 289, no. 1, pp. 49-55, 2003.

[27] J. A. Bartz, J. Zaki, N. Bolger, and K. N. Ochsner, "Social effects of oxytocin in humans: context and person matter," Trends in Cognitive Sciences, vol. 15, no. 7, pp. 301-309, 2011.

[28] A. D. Marshall, "Posttraumatic stress disorder and partnerspecific social cognition: a pilot study of sex differences in the impact of arginine vasopressin," Biological Psychology, vol. 93, no. 2, pp. 296-303, 2013.

[29] L. J. Young and Z. Wang, "The neurobiology of pair bonding," Nature Neuroscience, vol. 7, no. 10, pp. 1048-1054, 2004.

[30] A. J. Guastella, G. A. Alvares, I. B. Hickie, H. K. Chan, T. F. Chen, and R. B. Banati, "Unitary hormonal models, peripheral markers, and evaluation of response to drug: a response to Weisman and Feldman," Psychoneuroendocrinology, vol. 38, no. 5, pp. 627-628, 2013. 


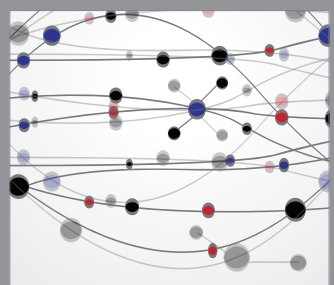

The Scientific World Journal
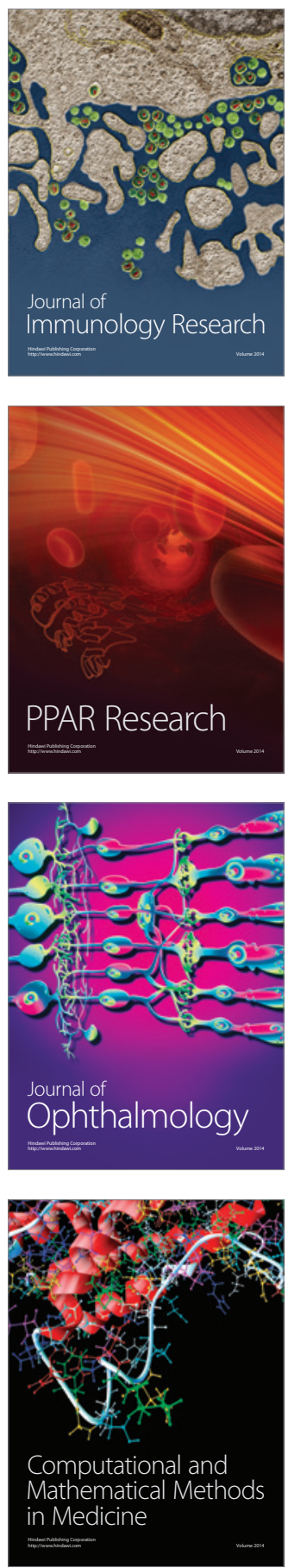

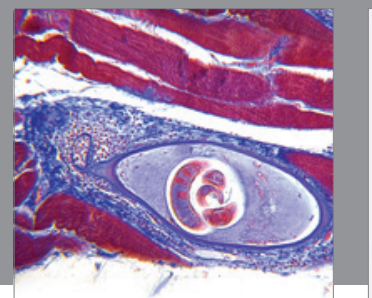

Gastroenterology

Research and Practice
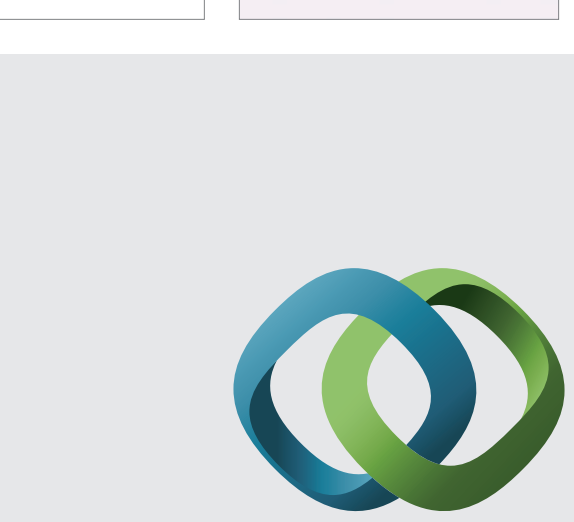

\section{Hindawi}

Submit your manuscripts at

http://www.hindawi.com
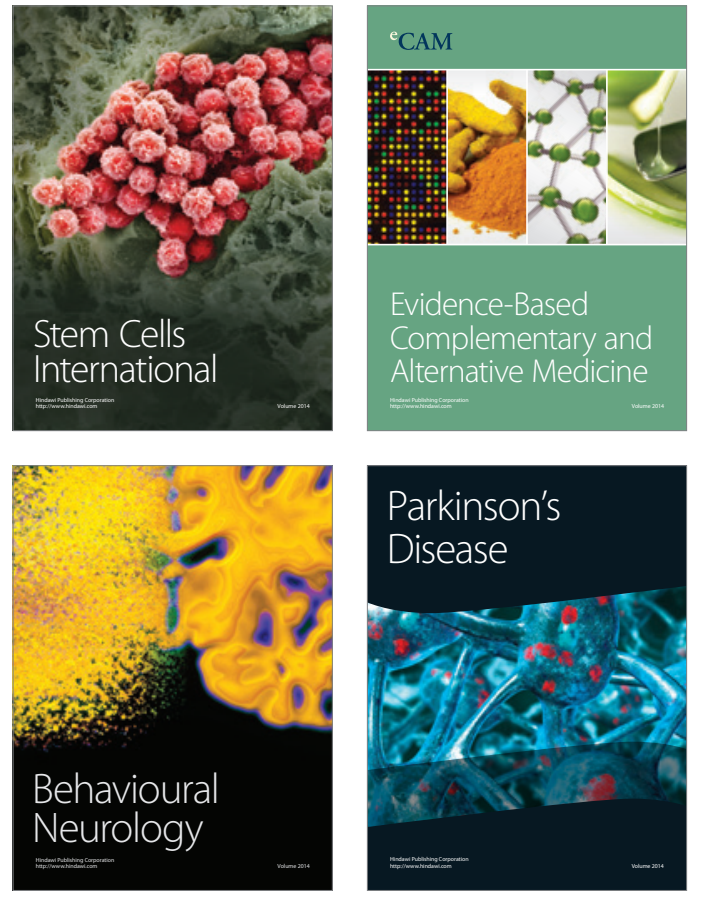
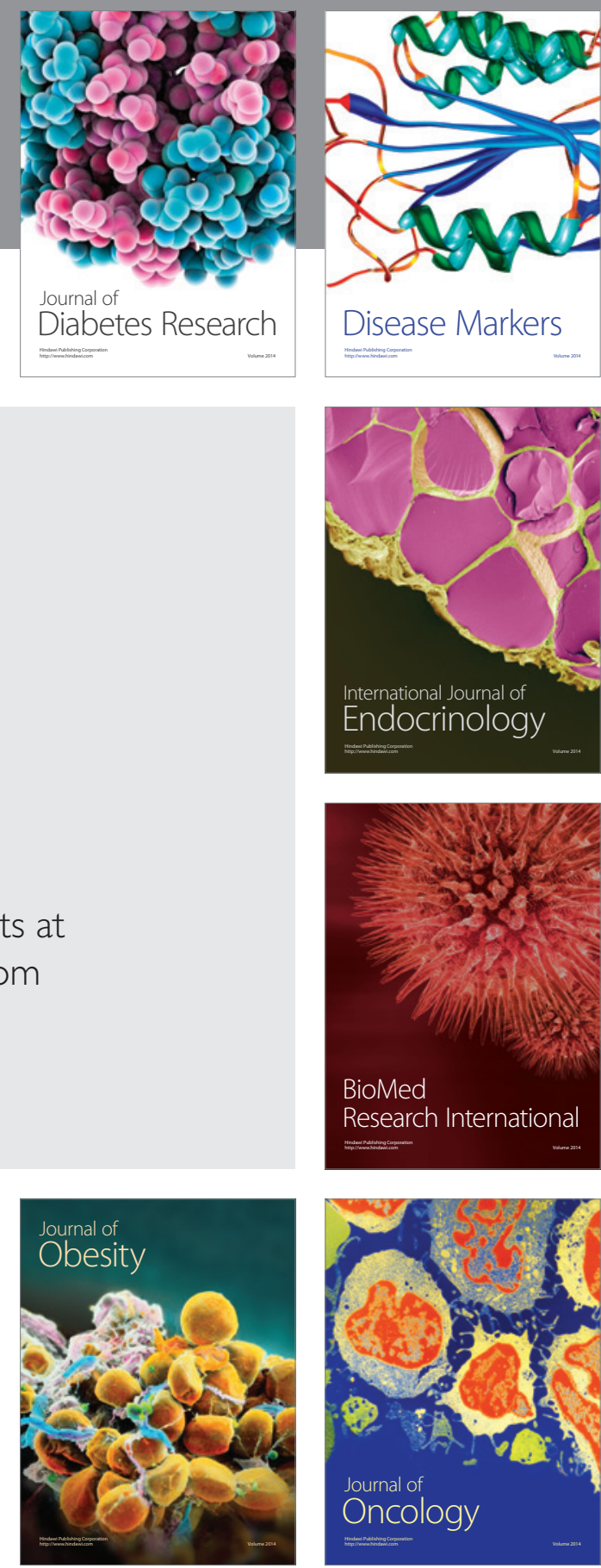

Disease Markers
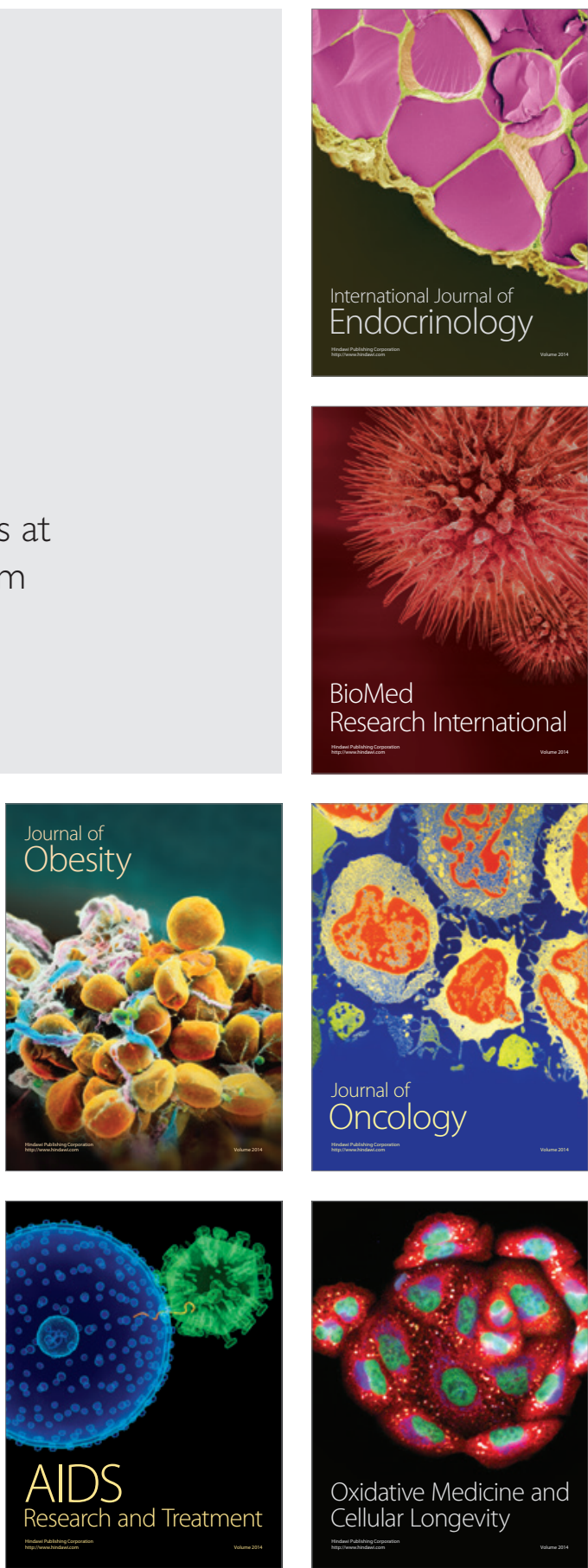